\title{
5.5 SYNCHROTRON RADIATION IN HIGH MAGNETIC FIELDS
}

\author{
D. F. FALLA and A. EVANS \\ Department of Physics, The University College of Wales, Aberystwyth, U.K.
}

\begin{abstract}
We suggest that if there exist within the Crab Nebula localised condensations of material containing high magnetic fields, ( $\gg 1 \mathrm{G})$, the rapidly evolving synchrotron radiation power spectrum emitted by a single electron can give a radiation continuum with a spectral index having a unique value similar to that observed in the optical and low-energy X-ray regions. One implication of this result is that a simple comparison between the observed fluxes of optical and gamma radiation emitted by the Nebula is no longer meaningful, so that one cannot draw any immediate conclusion regarding the fundamental mechanism of electron and gamma ray production.
\end{abstract}

The radiation continuum for the Crab Nebula in the near infra-red, optical and low-energy X-ray regions may be represented approximately by the power-law formula $F(v) \propto v^{-n}$, with $n \approx 1$, a characteristic feature that it shares with other radiation sources associated with energetic cosmic events.

It is generally agreed that the continuum arises from synchrotron radiation by relativistic electrons. The usual explanation for the spectral index $n$ is that it results from radiation, in a uniform magnetic field, by electrons that have an energy spectrum $N(E) \mathrm{d} E \propto E^{-\alpha} \mathrm{d} E$, where $\alpha=2 n+1$. We show here that for regions of high magnetic field, $n$ can be given a different interpretation, with the particular values $\frac{1}{2}$ and 1 having a special significance. We also consider the relevance of our arguments to the Crab Nebula.

The synchrotron radiation power spectrum, for an electron of energy $\gamma m c^{2}$ in a magnetic field $H$, is described by the characteristic frequency

$$
v_{c}=\left(\frac{3 e H}{4 \pi m c}\right) \gamma^{2}
$$

For $x$ defined as $v / v_{c}$, the power spectrum is given approximately by

and

$$
P(v) \propto H x^{1 / 3}, \text { for } x \leqslant \frac{1}{3},
$$

$$
P(v) \propto H \exp \left(-a x^{2 / 3}\right), \text { for } x>\frac{1}{3},
$$

where $a$ is a constant. The expression for the electron energy as a function of time,

$$
\gamma=\gamma_{0} /\left(1+\beta H^{2} \gamma_{0} t\right)
$$

where $\gamma_{0}$ represents the initial electron energy and $\beta$ is a constant, gives the radiation half-life for the electron as

$$
t_{1 / 2}=\left(\beta H^{2} \gamma_{0}\right)^{-1}
$$


The corresponding evolution of the synchrotron power spectrum can be represented by the variation of characteristic frequency with time,

$$
v_{c}=\left(v_{c}\right)_{0} /\left(1+t / t_{1 / 2}\right)^{2},
$$

where $\left(v_{c}\right)_{0}$ is the value of $v_{c}$ for $\gamma=\gamma_{0}$.

It is normally assumed that $v_{c}$ does not change appreciably over the relevant time interval, which is usually the period of observation, so that the radiation continuum can be derived from the synchrotron power spectrum by combining it with the electron energy spectrum. We examine here the situation for which the change in $v_{c}$ during the relevant time interval is appreciable. In Figure 1, $v_{c}$ has been plotted, from (5), for times that are comparable with the radiation half-life $t_{1 / 2}$.

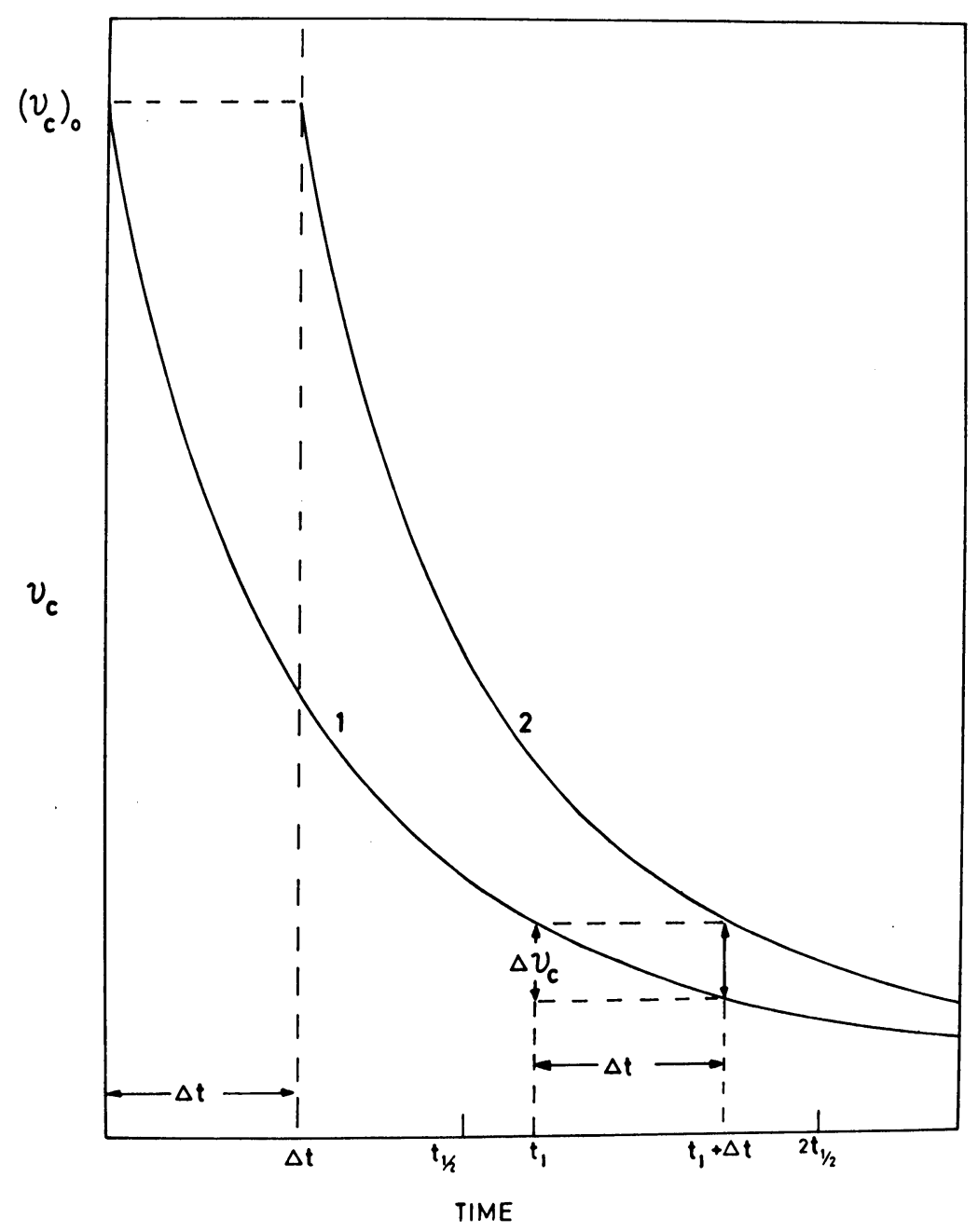

Fig. 1. Characteristic frequency as a function of time, for $t \sim t_{1 / 2}$. 
Suppose that at time $t_{1}$ there commences an observation of the radiation produced by a single electron, and that the observation lasts for an interval of time $\Delta t$; during this interval, a change in characteristic frequency $\Delta v_{c}$ occurs for the electron represented by curve 1 in the figure. For a system containing many electrons, a spread in characteristic frequency of the same magnitude $\Delta v_{c}$, for radiation observed at the instant of time $t_{1}+\Delta t$, may have been produced either by monoenergetic electrons with $v_{c}=\left(v_{c}\right)_{0}$ continuously injected into the field during the interval from $t=0$ to $t=\Delta t$; or it may have been produced by electrons injected at a time $t=\Delta t$, with a whole range of initial energies, represented by the difference in ordinates of curves 1 and 2 at this $t$ value.

For the case of the single electron observed over the interval from $t_{1}$ to $t_{1}+\Delta t$, we suggest that for the evolutionary effect to be regarded as significant, $\Delta t \gtrsim t_{1 / 2}$. This condition can also be written in the form

$$
H^{3 / 2} \Delta t \gtrsim \text { a constant, }
$$

the value of which depends upon the frequency of the observed radiation. In the radio region $\left(v_{c} \approx 10^{9} \mathrm{~Hz}\right)$, a magnetic field $\mathrm{H} \sim 10^{-4} \mathrm{G}$ gives $\Delta t \gtrsim 10^{6} \mathrm{y}$ : clearly, evolutionary effects are insignificant for a single electron radiating in this region of the spectrum. In the optical region however, $\mathrm{H} \approx 100 \mathrm{G}$ gives $\Delta t \gtrsim 10^{3} \mathrm{sec}$, a time comparable with photographic plate exposure times. In the X-ray region $\left(v_{c} \sim 10^{18} \mathrm{~Hz}\right)$, $H \approx 3 \mathrm{G}$ gives $\Delta t \gtrsim 200 \mathrm{sec}$, the detection time for $\mathrm{X}$-radiation from the Crab Nebula in the lunar occultation experiment of Bowyer et al. (1964). If therefore there exist regions of the Nebula containing high magnetic fields $H \gg 1 \mathrm{G}$, we may assume that, for a single electron, evolutionary effects are certainly significant for the $\mathrm{X}$-ray region, and probably for the optical region as well, for the periods of observation normally employed.

In the case of a system containing many electrons, $\Delta t$ can be given an alternative interpretation, as already indicated. In the Table I below, we show values of $\Delta t$ calculated from (6) for two commonly assumed magnetic field values, for the X-ray, optical and radio regions.

If $\Delta t$ in interpreted as the time over which monoenergetic electrons have to be continuously injected into the field for the equivalent spread $\Delta v_{c}$ in characteristic frequency to be appreciable, then it is apparent that condition (6) could be fulfilled, even for these low magnetic fields, for electron injection times normally considered.

TABLE I

\begin{tabular}{llll}
\hline$H$ (Gauss) & \multicolumn{1}{c}{$\Delta t$ (years) } & & \\
\cline { 2 - 3 } & $\begin{array}{l}\text { X-ray } \\
\left(v \approx 10^{18} \mathrm{~Hz}\right)\end{array}$ & $\begin{array}{l}\text { Optical } \\
\left(v \approx 10^{15} \mathrm{~Hz}\right)\end{array}$ & $\begin{array}{l}\text { Radio } \\
\left(v \approx 10^{9} \mathrm{~Hz}\right)\end{array}$ \\
\hdashline $10^{-3}$ & $>1$ & $>50$ & $\approx 10^{4}$ \\
$10^{-4}$ & $>30$ & $\gtrsim 10^{3}$ & $\geq 10^{6}$
\end{tabular}


While in our calculations of the theoretical radiation continuum now to be described, we consider only the case of a single electron observed over the time interval $\Delta t$, a similar mathematical method would be applicable to sources containing many electrons, with $\Delta t$ given its alternative interpretation.

We consider a power spectrum of the form (2), in which $v_{c}$ changes with time according to (4) and (5): the evolutionary effect is illustrated in Figure 2. The total

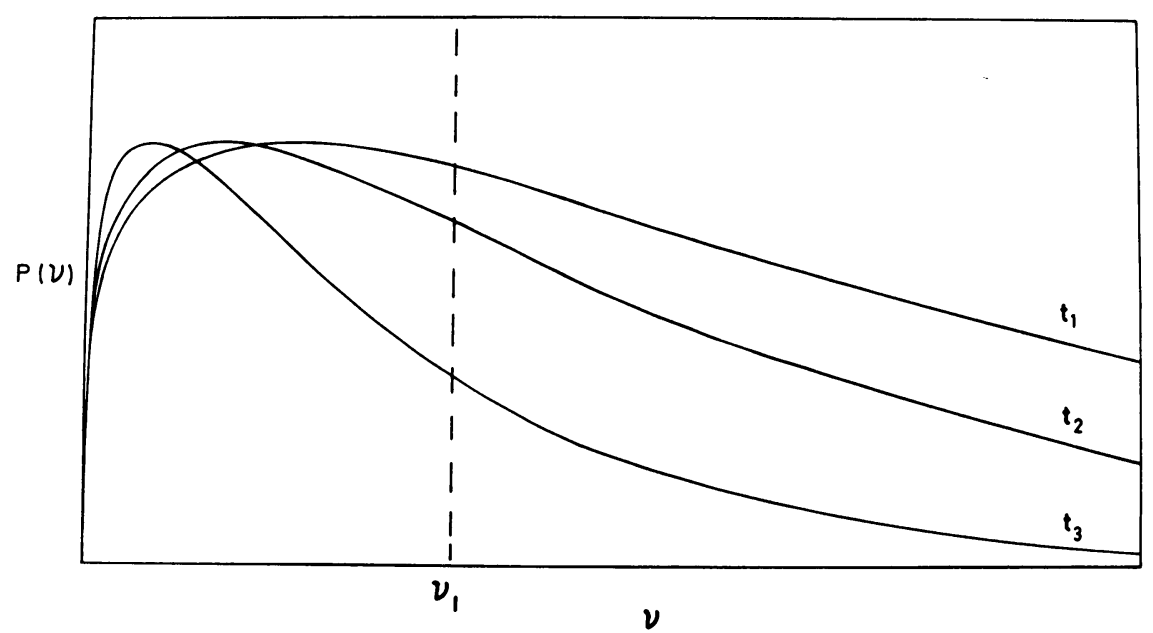

Fig. 2. Evolution of synchrotron radiation power spectrum, shown at successive moments of time $t_{1}, t_{2}$ and $t_{3}$.

radiation continuum produced by an electron of initial energy $\gamma_{0} m c^{2}$, radiating in a uniform magnetic field $H$, has been derived by taking the frequency $v$ as an independent parameter and, at each value of $v$, (for example, $v_{1}$ in the figure), computing the integral of the evolving power spectrum over the whole radiation lifetime of the electron. Figure $3 \mathrm{a}$ shows the radiation continuum for the optical and near infra-red regions, computed for one particular electron energy (given by $\gamma_{0}=75$ ), for several magnetic field values in the range from $2 \times 10^{4} \mathrm{G}$ to $10^{5} \mathrm{G}$; each curve gives the radiation continuum produced by a single electron.

If we perform the integration by an analytical method we find that, provided that the initial electron energy is sufficiently high, (a condition that can be expressed by the inequality $\log _{10} H \gamma_{0}^{2}>12,9$ and 3 for the $\mathrm{X}$-ray, optical and radio regions respectively), the integral can be written in the simple asymptotic form

$$
F(v)=2.63 \times 10^{-10}(v H)^{-1 / 2} \mathrm{erg} \mathrm{Hz}^{-1} .
$$

If the initial electron energy condition is not satisfied then this expression is only an approximation; in this case the more general formula used in deriving the curves of Figure 3a has to be taken. 


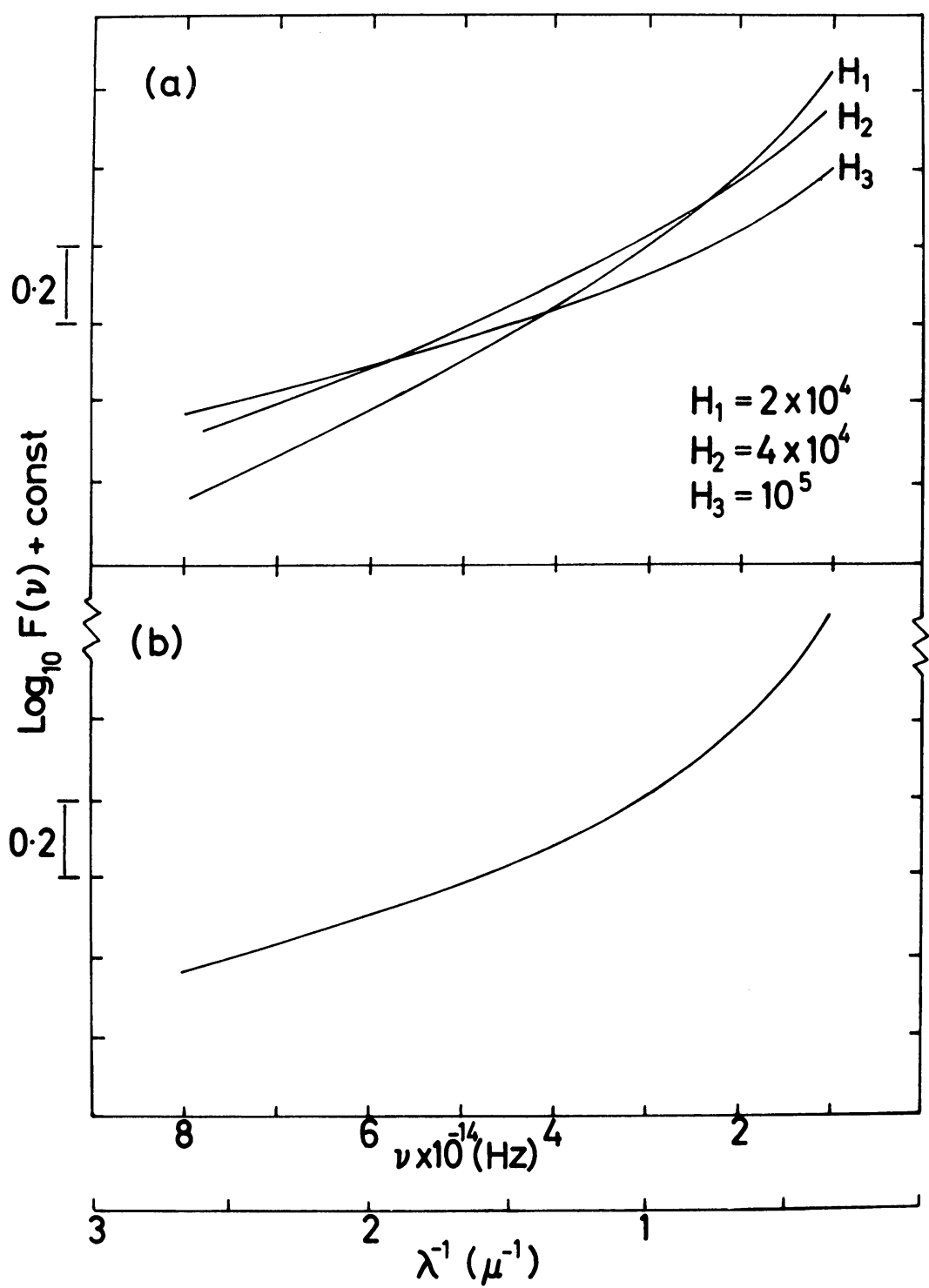

Fig. 3. $\quad F(v)$ as a function of $v$, (a) for $\gamma_{0}=75$ and three different $H$ values, (in gauss); (b) for $\gamma_{0}=75$ and a flat $H$ distribution.

It seems that the only requirements for the validity of the theory described above are that the electron should be confined to a uniform high magnetic field region, and that it should be observed for the whole of its radiation lifetime; if these requirements are fulfilled then a spectral index $n \approx \frac{1}{2}$ is obtained. Furthermore, the theoretical radiation continuum flux $F(v)$ at the frequency $v$ does not depend upon the initial energy of the electron: this has the important consequence that the spectral index for the 
radiation continuum is independent of the initial energy spectrum of the electrons injected into the magnetic field region.

For a complete radiation source it is necessary to integrate the computed radiation continua over an appropriate magnetic field distribution. We consider one particular case, - a flat distribution, in which all magnetic field values are equally probable. Inspection of the curves in Figure 3a reveals that for any frequency there exists an optimum $H$ value at which the computed flux $F(v)$ is a maximum; the locus of these maxima is the upper envelope of the curves in Fig. 3a, and is shown in Fig. 3b. We take the envelope plotted in Fig. $3 \mathrm{~b}$ as an approximate representation of the whole radiation continuum.

We find that for the optical and near infra-red regions, the envelope curve can be represented by the spectral index $n \approx 1$; we also find that an analytical method indicates that $n=1$ identically. We suggest that this value of $n$ has a special significance and arises in a manner quite different from that usually supposed: it could indicate that we are observing cosmic systems where different high magnetic field values occur with equal probability, and for which the radiating electrons have synchrotron power spectra that show a significant evolutionary effect.

High magnetic fields could be produced in localised condensations of material within the source. The radiating electrons either enter the magnetic field region from outside, or alternatively are generated in situ by the decay of pions produced in the nuclear interactions of protons incident upon the condensed material. (In low-energy pion decay, the electron energy distribution has a maximum at $\gamma_{0}=75$, the figure originally taken in our calculations). The rate of electron production in situ from a given proton flux is approximately proportional to the mass of the condensation, and does not depend upon the magnetic field contained by it; for the situation where the mass and magnetic field are completely uncorrelated, the suggested flat magnetic field distribution would be obtained.

We would expect this type of model to apply to all cosmic systems that contain high-energy protons together with condensations of material where there are high magnetic fields and where pion, and therefore electron, production can occur (Falla, 1970). A characteristic form for the radiation continua for sources associated with a wide variety of energetic cosmic events, including the Crab Nebula, has indeed been pointed out by Searle et al. (1965).

For the Crab Nebula in particular, our model has one clear implication: this is that the observed spectral index $n \approx 1$ is simply the characteristic value, the theoretical derivation of which we have described, so that it is not possible to obtain from the index any information concerning the energy spectrum of the interacting protons. Previously, comparisons between the synchrotron and gamma radiation fluxes had been made, with the object of testing the hypothesis that the relativistic electrons and gamma-ray photons arise from the decay of charged and neutral pions. In conventional synchrotron radiation models, where an electron energy spectrum with index $\alpha$ is involved, one can show that, for a given proton energy spectrum, the spectral index for the synchrotron radiation is exactly half that for the gamma radiation. For 
magnetic fields of magnitude $\mathrm{H} \sim 10^{-4} \mathrm{G}$, calculations by Gould and Burbidge (1967) led to the conclusion that, in view of the low upper limit to the gamma-ray flux, observed by Fruin et al. (1964), the pion production process could not be responsible for the electrons that radiate in the optical to low-energy $\mathrm{X}$-ray regions. On our model, however, where much larger fields are assumed to be present, the gamma radiation spectral index, which is determined by the spectral index for the interacting protons, is completely unrelated to the unique value $n=1$ for the synchrotron radiation: a straightforward comparison between the fluxes of synchrotron and gamma radiation is therefore no longer meaningful.

We consider also the radiation emitted by the Crab pulsar, NP 0532. Electrons radiating in a region of uniform magnetic field of magnitude $\gtrsim 10^{6} \mathrm{G}$, even greater than the values we have assumed for the Nebula itself, would very probably, according to our arguments, be observed over the whole of their radiation lifetimes. For electrons with $\gamma \lesssim 10^{4}$ our model, which is based on standard synchrotron radiation theory, would still be applicable; for electrons with higher energies, however, it is probable that the synchrotron emission would be affected by strong radiation damping (Shen, 1970). An interpolation of the observed radiation continua for this pulsar between the optical and X-ray regions shows evidence (Trümper, 1970) for a spectral index $n=\frac{1}{2}$. We may note that the modification of the standard synchrotron radiation theory that we have discussed here would give this value of $n$ for a single condensation of material with which a uniform high magnetic field is associated.

\section{References}

Bowyer, S., Byram, E. T., Chubb, T. A., and Friedman, H.: 1964, Science 146, 912.

Falla, D. F.: 1970, Astrophys. Letters 6, 77.

Fruin, J. H., Jelley, J. V., Long, C. D., Porter, N. A., and Weekes, T. C.: 1964, Phys. Letters 10, 176.

Gould, R. J. and Burbidge, G. R.: 1967, Handbuch der Physik 46 (2), 265.

Searle, L., Rodgers, A. W., Sargent, W. L. W., and Oke, J. B.: 1965, Nature 208, 1190.

Shen, C. S.: 1970, Phys. Rev. Letters 24, 410.

Trümper, J.: 1970, Astrophys. Letters 5, 271. 